Instituto Internacional de Investigación y Desarrollo Tecnológico Educativo INDTEC, C.A.

DOI: https://doi.org/10.29394/Scientific.issn.2542-2987.2019.4.13.6.114-137

OAI-PMH: http://www.indteca.com/ojs/index.php/Revista Scientific/oai

Artículo Original / Original Article

\title{
Desarrollo Cognitivo de los Estudiantes en Complejos Virtuales Educativos en Venezuela desde la Perspectiva Filosófica
}

\author{
Autores: Jurisbel Andreina Pérez Jiménez \\ Universidad Nacional Experimental "Rafael María Baralt", UNERMB \\ jurisbel357@hotmail.com \\ Zulia, Venezuela \\ https://orcid.org/0000-0003-0345-0042 \\ Jurismar Ivana Pérez Jiménez \\ Universidad Nacional Experimental "Rafael María Baralt", UNERMB \\ jurismarperez@gmail.com \\ Zulia, Venezuela \\ https://orcid.org/0000-0002-8866-6215 \\ Blanca Leticia Caldera de Sánchez \\ Universidad Nacional Experimental "Rafael María Baralt", UNERMB \\ blancacaldera@hotmail.com \\ Zulia, Venezuela \\ https://orcid.org/0000-0002-4198-6748 \\ Luisa Asención Serra López \\ Universidad del Zulia, LUZ \\ serra sl@yahoo.com \\ Zulia, Venezuela \\ https://orcid.org/0000-0002-7561-2541
}

\section{Resumen}

La educación en Venezuela ha sido fuertemente criticada y con ella los modelos educativos, dado al desarrollo cognitivo y colonizado que se viene presentando en los estudiantes universitarios frente a los entornos o complejos virtuales de aprendizaje. ¿Pero cómo se da este fenómeno desde la perspectiva filosófica? En este sentido, esta investigación tuvo como objetivo, analizar la vinculación entre el desarrollo cognitivo de los estudiantes y los complejos virtuales describiendo la perspectiva filosófica que se encuentra presente en ello, estudiando el comportamiento humano frente a los cambios políticos, económicos y sociales. Desde la perspectiva social se contempla la educación moderna frente al impacto tecnológico. El tipo de investigación fue de tipo documental descriptiva, resultando que las tendencias filosóficas en los complejos virtuales para el desarrollo cognitivo se encuentra presente en el conocimiento empírico que involucra la ontológica de realismo, la dialéctica, la hermenéutica y el constructivismo y en conclusión se constituye como la manera idónea para vincular los criterios necesarios que permiten implementar los complejos educativos virtuales, fusionando la innovación con aspectos relativos a la enseñanza y el desarrollo humano.

Palabras clave: cognición; tecnología educativa; filosofía educativa.

Fecha de Recepción: 30-10-2018
Fecha de Aceptación:

06-03-2019

\section{Fecha de Publicación: 05-08-2019}




\title{
Cognitive Development of Students in Virtual Educational Complexes in Venezuela from the Philosophical Perspective
}

\begin{abstract}
Education in Venezuela has been strongly criticized and with it the educational models, given the cognitive and colonized development that has been presented in the university students in front of virtual environments or learning complexes. But how does this phenomenon occur from a philosophical perspective? In this sense, this research aimed to analyze the link between the cognitive development of students and virtual complexes describing the philosophical perspective that is present in it, studying human behavior in the face of political, economic and social changes. From the social perspective, modern education is considered in the face of technological impact. The type of research was descriptive documentary type, resulting that the philosophical tendencies in the virtual complexes for the cognitive development is present in the empirical knowledge that involves the ontological of realism, the dialectic, the hermeneutics and the constructivism and in conclusion it is constituted as the ideal way to link the necessary criteria that allow implementing the virtual educational complexes, merging innovation with aspects related to teaching and human development.
\end{abstract}

Keywords: cognition; educational technology; educational philosophy.

Date Received: 30-10-2018
Date Acceptance:

06-03-2019
Date Publication:

05-08-2019 


\section{Introducción}

La educación en Venezuela ha venido sufriendo los estragos de la crisis presupuestaria y con el pasar de los años se ha agudizado la situación y vuelto insostenible mantener un estatus educativo que realmente de respuesta y aporte verdaderamente a la sociedad, sumado al nivel de pobreza y desinterés de los estudiantes universitarios que cada día se ausentan de las casas de estudio privadas y autónomas optando por un futuro que resuelva la necesidad del momento y no del futuro. En este sentido, se vuelve difícil atender los estándares institucionales a fin de garantizar una educación incluyente en aras de fortalecer e incrementar las posibilidades de los egresados a un ambiente laboral no excluyente abocado a las tecnologías de información y comunicación del futuro apostando a una educación transformadora, critica y reflexiva.

De lo anterior, se puede profundizar que dentro del contexto educativo universitario se encuentra inmersa la filosofía como ciencia epistemológica llevada al constructivismo como corriente de pensamiento y se fundamenta en las ideas a priori comprendidas como juicios sintéticos, analítica y dialéctica trascendentales que reflejan el carácter unificador del espíritu humano. De acuerdo con Londoño (2008a): "el constructivismo está caracterizado por su vinculación con la psicología cognitiva y la epistemología histórica hace referencia a una nueva época, en la que predomina el aprendizaje inteligente y las estructuras de racionalidad, desde su desarrollo" (pág. 75).

En este apartado establece Piaget (1972), citado por Raynaudo y Peralta (2017): que "las estructuras a priori no son preestablecidas, sino que se desarrollan progresivamente" (pág. 142); asimismo, expresa Kant (1938), citado por Zamudio y Castorina (2017), que el mecanismo priori: "en el tiempo ninguno de nuestros conocimientos preceden a la experiencia (...) Pero si es verdad que todos nuestros conocimientos comienzan con la experiencia, todos, sin embargo, no proceden de ella" (pág. 9); esto se debe a la 
incorporación de ideas de desarrollo y progresión, caminando hacia la construcción del conocimiento. Acorde con Londoño (2008b): el constructivismo sostiene que "sólo entendemos lo que podemos construir o reconstruir" (pág. 76); significa el retorno a la experiencia del individuo.

Es por esto, que los complejos virtuales educativos se muestran como una alternativa innovadora de aprendizaje que se aboca a la transformación e inclusión de diferentes mecanismos y herramientas de interacción estructurados y moldeados a modelo constructivista desde entornos virtuales enmarcados en el diseño y producción de los contenidos arquitectónicos del portal, de navegación y de interfaz. Expresa Bustos y Coll (2010): que los entornos virtuales cuentan con un "sistemas de administración de contenidos" (pág. 168); abarcando todos los recursos digitales sujetos a la web. Aquí juega un papel importante el registro del dominio web, el servicio de hosting, los servidores, la banda de transferencia y el sistema de pagos electrónicos.

Cabe destacar, que dentro del portal educativo se encuentran las famosas aulas virtuales que hacen comunicación directa institucional con los estudiantes además de que promueve la capacitación permanente de los facilitadores y estos a su vez emplean metodologías que buscan afianzar el conocimiento permanente y por descubrimiento. Expone Camacho (2009), citado por Flores y Bravo (2012): que "una de las metodologías más reconocidas es la PACIE la cual busca propiciar una explosión de información a través de la fase Presencia, Alcance, Capacitación, Interacción e E-learning" (pág. 9).

Tomando como referencia lo anteriormente expuesto, se concibe que el desarrollo cognitivo o cognoscitivo se encuentra presente en los entornos virtuales de aprendizaje por seguir la corriente filosófica, al perseguir la vertiente constructivista vista del conocimiento perceptivo o por la teoría del psico-génesis o psicología genética, el cual se basa en el proceso cognitivo se da al existir una relación entre el sujeto que conoce y el objeto que será 
conocido reconociendo un fenómeno que da paso al estallido del conocimiento.

Estas tendencias en los complejos virtuales para el desarrollo cognitivo se fundamentan en el aprendizaje complejo, su evaluación y fomento debido a que intervienen múltiples procesos y rasgos que determinan y condicionan el comportamiento combinando categorías heterogéneas, además de que toma tiempo construirlo, es multicausado, donde intervienen variables de diferente naturaleza, añadiendo características del contenido que se aprende, de modo que para Guanoluisa (2016):

considerando el desarrollo evolutivo del ser humano este ha experimentado una serie de cambios en su estructura genética y desarrollo intelectual a través de las habilidades cognitivas que desarrollan de acuerdo al perfeccionamiento de las destrezas con criterio de desempeño a través de las prácticas experiméntales (pág. 17).

En este sentido, la perspectiva filosófica orienta al ser humano a ser explorador de su propio conocimiento a abocarse a las nuevas tecnologías de enseñanza aprendizaje que apuesten a una educación diversificada y no globalizada que busque sistematizar los procesos educativos de forma dinámica y creativa con puertas a una inteligencia capaz de interactuar siendo participe de fenómenos críticos tomando herramientas tecnológicas que nos acerquen a un sistema mucho más desarrollado y que incentive a través del conocimiento subyacente; buscando fusionar la innovación con aspectos relativos de la enseñanza y el desarrollo humano involucrando el conocimiento empírico derivado de la corriente constructivista. Además de ser un aporte documental a futuras investigaciones.

\section{Referentes teóricos}

Para Briones (2002a): existen grandes escuelas filosóficas, las cuales son "aquellas que estudian la naturaleza de los objetos, las posibilidades y los 
niveles del conocimiento que se pueden lograr. También contienen diversas

hipótesis ontológicas y gnoseológicas en la que se encuentran diferentes concepciones del mundo" (pág. 22).

El materialismo, trata de una doctrina según la cual todo lo que existe es materia. El pensamiento y los fenómenos mentales se desarrollan a partir de la materia y deben ser explicados en términos físicos. El materialismo dialéctico tiene como una de sus preocupaciones centrales el cambio de la realidad las leyes de la dialéctica, según Kant (1922), citado por Londoño (2008), son:

a) La ley de transformación de los cambios cuantitativos en cualitativos; b). La ley de interpenetración de los opuestos; y c). La ley de la negación, la cual demanda una situación dada que luego es reemplazada por otra que también es negada (pág. 45).

El racionalismo, se considera como el metafísico debido a que es de carácter racional y psicológico dado a lo emocional y a la voluntad. Este afirma que es posible conocer la realidad mediante le pensamiento puro, sin necesidad de ninguna premisa empírica. El conocimiento es propiamente tal cuando tiene necesidad lógica y validez universal. Por su parte, Kant (1922), citado por Londoño (2008): expresa que sólo "la razón puede permitir decir que una cosa es como es y no puede ser de otra manera" (pág. 45).

El empirismo, de acuerdo con el Diccionario soviético de filosofía (1965): proviene del griego empeiria que significa experiencia (pág. 137); situación que se opone totalmente al racionalismo, expone Kant (1922), citado por Londoño (2008): que "es un conocimiento que proviene en gran medida, de la razón. Para el empirismo radical, la mente es como una tabla rasa que se limita a registrar la información que viene de la experiencia" (pág. 45).

El realismo, se distingue como metafísico, para Briones (2002b): tiene su "existencia real, independiente de ser pensadas o no" (pág. 25); es decir, 
los objetos percibidos por los sentidos, tienen una existencia independiente del propio ser percibido. Estas representan todo tipo de conocimiento que tiene el valor de verdad.

El idealismo, Briones (2002c): viene dada "a la conciencia, a las ideas, al pensamiento, al sujeto, al yo, en el proceso del conocimiento" (pág. 26); en este sentido, tal corriente filosófica contradice la existencia de objetos que sean independientes de la conciencia, pensamientos o ideas.

El positivismo, según Briones (2002d): se basa en la "experiencia sensible" (pág. 28); es una doctrina que promueve la exaltación de los hechos contra las ideas y las ciencias experimentales sobre las teóricas, este proceso es únicamente posible a través conocimiento sólo es posible con la observación y el experimento.

Según Briones (2002e), el positivismo lógico parte de la concepción del conocimiento por medio de la experiencia comprobada o verificada a través de los sentidos (pág. 30); apoyado en los recursos de la lógica moderna, por lo tanto, las hipótesis primarias, enunciados singulares, o totalmente libres de prejuicios mentales, pueden ser considerados como científicos solo si son sometidos a la lógica y planificación empírica, y los que no son sometidos a la lógica son considerados sin sentido o absurdos.

Asimismo, existen otras escuelas filosóficas que sirven de apoyo indirectamente a la construcción de las ciencias sociales, estas son:

La fenomenología: tal doctrina fue desarrollada por Edmund Husserl (1859-1938), y se denomina ciencia, porque estudia la aproximación de los hechos o fenómenos, es decir, el ámbito donde se hace presente esta realidad, esta consiste en la búsqueda de la comprensión basada en lo que es obvio, sencillamente porque es la realidad percibida. La idea fundamental según Briones (2002f): consiste en la intuición empírica de las cosas tal y como se manifiesten, analizando e interpretando según su experiencia (pág. 31). 
La filosofía analítica del lenguaje de Wittgenstein, su principal fundador es Ludwig Wittgenstein (1889-1951), y parte de la premisa, que todo lo que puede ser pensado puede expresado por el lenguaje. De acuerdo con Briones, (2002g):

el lenguaje tiene como propósito principal establecer hechos, para lo cual necesita hacer una figura de ello, proceso que implica establecer una correspondencia entre el plano de lo real y el plano lingüístico, de manera que, es necesario comprender como funciona, sobre todo, como deben ser usadas (pág. 33).

Expone Briones (2002h), que la hermenéutica es: "una de las primeras aproximaciones a la hermenéutica actual la hizo Wilhelm Dilthey (1833-1911) para quien esta disciplina debe ser el método de las ciencias del espíritu" (pág. 36); en este sentido, considera importante todo aquello que puede ser captado por la vivencia, a fin de describir lo vivido, interpretándolo de modo que, tenga un significado y sea comprensible.

\section{Entornos Virtuales de Aprendizaje}

Son complejos enriquecidos, nutridos del conocimiento colaborativo, manifiestan Serrano y Pons (2011): que se rige bajo un paradigma constructivista, siendo este "un proceso de construcción genuina del sujeto" (pág. 3); estos entornos e-learning funcionan como sistemas de gestión y autogestión, ofreciéndole al docente todas las bondades de una clase tradicional, asimismo señala Uribe (2017): que se desarrolla en los estudiantes, gran autonomía y autogestión de su propio aprendizaje (pág. 3).

\section{Características de los EVA}

Estos espacios tienen un conjunto de características que to hacen atractivo a la comunidad en general, todos estos aspectos positivos hacen el espacio satisfactorio para los participantes, para Mestre, Fonseca y Valdés 
(2007), poseen los siguientes atributos:

Hipermedia: combina el hipertexto con los multimedia de manera que la información digital -presentada en diferentes formatos- se distribuye a través de enlaces de hipertexto; Telepresencia: se debe a las sensaciones a las que da lugar la participación en el entorno hipermedia y a la posibilidad de relacionarse con otras personas que también acceden a él; Navegación en red: el usuario en su exploración por la red puede elegir con gran libertad la información que desee dispuesta en el espacio, con interacción controlada y centralizada; Interacción bidireccional, interpersonal y equipo $y$ los contenidos: el participante puede tener comunicación directa con el profesor, con sus compañeros, e incluso con el contenido multimedia (págs. 20-21).

\section{Proceso de selección de un entorno virtual de aprendizaje}

Ante un proceso de selección, es necesario delimitar y establecer la metodología pedagógica a implementarse en la modalidad e-learning $\circ b$ learning, seguidamente las actividades que se tienen previstas desarrollar para el alcance de las competencias y finalmente, adecuar la herramienta de acuerdo a las expectativas de los involucrados.

Es por esto, que es importante establecer estándares de calidad que permitan valorar el desempeño de las plataformas disponibles en el mercado en pro de la formación integral del estudiante en sus diferentes áreas de conocimiento.

6. Dentro de las generalidades en las plataformas e-learning, se encuentran las siguientes:

\subsection{Características técnicas}

Esta, se encuentra constituido por las cualidades que a nivel de software ofrece el sistema al usuario:

1. El tipo de licencia: el cual puede ser privada o gratuita.

2. El idioma: ya que cuentas con soporte multi-idioma. 
3. El sistema operativo y tecnología empleada: dado a que combina la compatibilidad de los recursos a el sistema.

4. La documentación: porque ofrece manuales de ayuda para comprensión y correcta aplicación de las herramientas que plataforma provee.

5. La comunidad: está constituida por las comunidades de apoyo que aportan a la construcción de conocimiento de forma cooperativa o colaborativa, en pro de un bien común, y estos son los usuarios y técnicos.

\subsection{Características pedagógicas}

Consiste en los recursos o herramientas que contribuyen al desarrollo dinámico de una clase virtual, en sentido las plataformas deben estar en capacidad de permitir:

1. La gestión y administración de las tareas que debe realizar el estudiante.

2. La interacción entre los involucrados.

3. El diseño, desarrollo e implementación de contenidos adaptados al contexto educativo.

4. La evaluación, ejecución y seguimiento de estrategias colaborativas.

5. Y, por último, que el estudiante tenga la oportunidad de personal el entorno.

En tal sentido, La Metodología PACIE, se ha estructurado con el fin de permitir desarrollar de forma lógica y sistematizada todos los momentos implícitos de una clase tradicional, pero enfocada en un aula virtual, según Camacho (2009), citado por Flores y Bravo (2011): "se debe distribuir en varias etapas las cuales son el bloque 0 o PACIE, el bloque académico, el bloque de cierre" (pág. 85); es por lo que su creador, el Ing. Pedro Camacho, describe 
cada uno, de la siguiente manera:

EI Bloque PACIE o bloque 0: Muestra información general sobre el curso, el tutor y los procesos de evaluación, recursos o actividades para conocer el aula, quien es el facilitador porque lleva el proceso tutorial; El Bloque Académico o Sección de Exposición: Que posee la información y contenidos en sí de la materia, cátedra o asignatura, los documentos que se quiere compartir, los enlaces hacia los cuales se desea diversificar y la exposición temática que deseemos realizar; El bloque de cierre o Sección de Negociación: Ayuda a no dejar cabos sueltos dentro del aula, a culminar actividades pendientes, a cerrar procesos inconclusos, a negociar desacuerdos en evaluaciones, a retroalimentarse con la opinión de los estudiantes, no solo para los contenidos y estructura del aula en sí, sino para la labor tutorial (págs. 85-87).

\section{Plataformas de Código Abierto "open source", para el desarrollo de aulas virtuales de aprendizaje}

Existen variedad de plataformas que se encuentran en el mercado web de forma gratuita, dado a que forman parte de la selecta familia del software libre y gracias a los privilegios para su uso, edición y distribución pudiera ser adaptado conforme a las necesidades del área o contexto al cual se quisiera contribuir, entre los más destacados tenemos: ATutor: Sistema de Gestión de Contenidos de Aprendizaje; Chamilo LMS: Plataforma de E-learning de software libre; Claroline: Plataforma de aprendizaje y software colaborativo de código abierto; Docebo: plataforma para e-learning, también conocido como aprendizaje de manejo de sistema; Dokeos: Entorno de educación en línea y una aplicación de administración de contenidos de cursos; LRN: Aplicación open-source para las comunidades de aprendizaje e investigación; FLE3: Medio de aprendizaje basado en la web o un ambiente de aprendizaje virtual; y Moodle: herramienta de gestión de aprendizaje, o más concretamente de Learning Content Management. 


\section{Dificultades a las que se enfrentan las aulas virtuales de aprendizaje}

La enseñanza a través de las aulas virtuales, genera experiencias que pudieran obstaculizar el proceso de aprendizaje, tal como sucedió en un estudio en nueve universidades de Andalucía, según los autores Rojas, Pérez, Torres y Peláez (2014): coincidieron que la formación de los docentes en estas tecnologías, se ha centrado fundamentalmente en la autoformación, en la formación de software básicos (pág. 238).

Asimismo, en la preparación del aula y el contenido didáctico del aula es vista como una carga extra del docente, y en la mayoría de los casos queda solo delimitada a una persona que tiene formación en Moodle y áreas comunes. También se observa la reutilización de actividades dispuestas en la web, es decir, no existe una creatividad que promuevan y favorezcan el uso del espacio.

\section{Criterios a evaluar en los entornos virtuales de aprendizaje}

Existen tres aspectos básicos para el análisis de la calidad de la formación online realizada a través de los entornos virtuales de aprendizaje según Torres y Ortega (2003), citado por la Manriquez (2016), donde se enmarca la:

Calidad Técnica; caracterizada por la plataforma como producto de la solidez, estabilidad de los procesos de gestión y de enseñanza aprendizaje. Se encuentra también, la calidad organizacional y creativa, enfocada al diseño de instruccional, la posibilidad de adaptación y uso. La calidad comunicacional definida por la sincrónica y asincrónica de las personas involucradas y finalmente, la calidad didáctica orientada hacia la incorporación de actividades coordinadas metodológicamente basados en los principios de aprendizaje de las teorías conductistas, cognitivistas y constructivistas (pág. 1). 


\section{Metodología}

Esta investigación es de tipo documental, según Arias (2006): por ser un "proceso basado en la búsqueda, recuperación, análisis, crítica e interpretación" (pág. 27); de fuentes documentales acerca del estudio, al mismo tiempo descriptiva puesto que "consiste en la caracterización de un hecho, fenómeno, individuo o grupo, con el fin de establecer su estructura o comportamiento" (pág. 24). Por consiguiente, el presente artículo es producto de una investigación documental y descriptiva dado que para la construcción y argumentación del problema se empleó mediante la revisión de material bibliográfico y descriptivo porque se describe el fenómeno variable de estudio.

\section{Resultados}

Tomando como referencia el artículo publicado por Lander (1999): "¿Conocimiento para qué? ¿Conocimiento para quién?" donde reflexiona sobre la universidad y la geopolítica de los saberes hegemónicos (pág. 25); se puede establecer una conexión entre el papel que denota el desarrollo cognitivo de los estudiantes universitarios con respecto a los complejos virtuales educativos en Venezuela desde la perspectiva filosófica dado a que se pueden ejemplificar varias posturas que a nivel mundial atacando a la sociedad desde el punto político, social y educativo.

Es el caso de la globalización frente a la sociedad del mercadeo, la cual está sufriendo dificultades en cuanto al déficit de irregularidades del capital y de inversión tecnológica; provocando una deserción en cuanto a una mejor calidad educativa. Además, de que la lucha de la reflexión crítica está por encima del pensamiento genuino, con el objetivo de indagar otras áreas de conocimiento, enmarcadas en lo práctico y en las vivencias del sujeto, sumada a las oportunidades sociales de cara a la hegemonía del capital.

De modo que, las universidades latinoamericanas poseen disciplinas donde se practica el parcelamiento burocrático de los saberes, y privatizan la 
búsqueda de soluciones ante los problemas. Estructuras que se abocan a la normalización, a los aspectos científicos de transformación visionaria enfocada a las organizaciones occidentales del mundo, trabajando así a través de la descolonización del conocimiento y al bloqueo metodológico frente a la imposición de la investigación empírica, la cual puede ser cuantificada, posee una rigidez científica, e invalida la reflexión universal, no permite la inclusión de juicios o reconocimientos, incluye el modelo tecnológico actual, y propone la participación de la sociedad y el incentivo de oportunidades a futuro.

\section{Conclusiones}

Es evidente que las ciencias sociales a través de las teorías del conocimiento filosóficos son fundamentales en el desarrollo del intelecto humano desde el punto de vista cognitivo, social y cultural tomando como referencia que las experiencias proporcionan evidencias que contribuyen a la transformación del comportamiento.

En este sentido, nacen las diferentes corrientes o tendencias filosóficas tales como: el racionalismo, el materialismo, el realismo, el empirismo, el idealismo, el positivismo, el positivismo lógico y otras tantas que aportan como la fenomenología, la filosofía analítica del lenguaje de Wittgenstein y la hermenéutica, todas ellas van en pro de estudiar el proceder humano, y de anticipar los mecanismos de defensa ante el nuevo aprendizaje.

Cabe destacar, que la realidad es percibida de manera diferente según los prejuicios que la mente humana de forma preestablecida y este está vinculado con la ontológica de realismo, la dialéctica, la hermenéutica, el constructivismo, que cuestiona el ideal de racionalidad moderno; asumiéndose que la objetividad da posición a un sujeto trascendental por naturaleza reconociéndolo como una entidad independiente del objeto; partiendo que la cognición no tiene límites epistemológicos, pero si se encuentra frente a la crítica de la racionalidad moderna, mientras que una forma subjetividad resulta 
del proceso de la cognición donde se demuestra la verdad y se traduce en una construcción, un sentido, una creencia, una dialéctica de lo relativo, entre otros. En cambio, cuando se trata de una verdad objetiva la ciencia es la responsable de producirla.

Visto así, desde el punto de vista práctico de la perspectivas filosóficas, estas apuntan a un pensamiento crítico ya que debela el desafío como resultado de las posturas de cada profesor o facilitador de enseñanzaaprendizaje abocados a descubrir alternativas que denoten enfoques absolutos convencionales del conocer y el vivir en el entorno que hoy en día nos desenvolvemos; situándonos en la percepción de la posibilidad y en la necesidad de construir futuros facultativos y que mejoren la calidad de vida del ser humano.

En efecto, el empirismo como corriente epistemológica está enfocado al aprendizaje del sujeto desde su experiencia, adoptando el aspecto psicológico, racional, gnoseológico y metafísico que hacen vida en el conocimiento empírico y que contribuyen verdaderamente al pensamiento humano, promoviendo la transformación y el método analítico, frente a las ideologías y prácticas convencionales.

Sin duda, el desarrollo cognitivo presente en los estudiantes, se puede contextualizar dentro del movimiento empírico el cual esquematiza que este, se encuentra presente en las diferentes actividades que se realizan como: investigar, aprender a aprehender, reflexionar, analizar, sintetizar, reflexionar, criticar, entre otros; en consecuencia, el individuo corrige y mejora la calidad y efectividad con que realiza sus procesos acondicionando sus pensamientos.

En conclusión, el empirismo como disciplina filosófica se fundamenta desde la actitud contradictoria, dictaminando que la experiencia es crucial y los cambios conductuales son relevantes para el logro de los objetivos. Los esquemas mentales y el proceso de acomodación y reacomodación se articulan para aprender a aprehender y coaccionan para lograr un aprendizaje 
significativo que obligue al individuo, como ser pensante a fijarse metas. El conocimiento es el mecanismo de defesa ante lo desconocido y el aprendizaje representa el proceso para lograrlo; asumiendo esto como verdad absoluta se considera que la psicológica y epistemológica precede únicamente de la experiencia, ya sea experiencia por reflexión o por sensación, y que esta es su única base, y desde esta corriente filosófica el empirismo se convierte en eje central para el aprendizaje continuo que realmente se encuentra internalizado en el individuo.

En cuanto a la relación que existe entre el desarrollo cognitivo y los complejos educativos virtuales dentro de la corriente epistémica, es que esta desplaza la razón por la sensibilidad y demuestra que el conocimiento sensible es el único conocimiento válido porque se inspira en los hechos, y en oposición a las utopías teóricas, como a las fantasías y a las interpretaciones especulativas que hablan sobre el conocimientos innato; es decir, que en este caso el estudiante ya supone tener facultades para hacer alguna actividad y no son resultados de la experiencia; principios que no son válidos porque el empirismo según John Locke (1632-1704), citado por Barrionuevo (2005): se adquiere a través del uso de las facultades innatas, que limita la posibilidad de experimentar, asociado a la metafísica que promueve la desconfianza en el conocimiento cognoscitivo (pág. 3).

En este sentido, se muestra el Entorno Virtual de Aprendizaje (EVA) o por sus siglas en inglés, Virtual Learning Environment (VLE) como espacios en la web, que contienen un conjunto de herramientas y recursos informáticos que posibilitan la interacción unidireccional, bidireccional y multidireccional. Moodle es una herramienta que permite la gestión del aprendizaje, siendo este uno de los entornos virtuales de aprendizaje más reconocido, con licencia gratuita, tiene dominio web propio, plataforma de Aulas Virtuales con imagen corporativa propia, módulos educativos incorporados, propicia la capacidad para los estudiantes y actividades educativas reales, tiene sistema e-mail 
institucional, módulo de telecomunicaciones masivas para estudiantes y padres de familia, recursos para la creación de un periódico digital, capacidad de inclusión de videos, galería fotográficas y matriculación en línea, capacidad de cobros electrónicos con tarjeta de crédito y talleres presenciales para el personal docente y administrativo de la Institución.

Este segundo implica el compromiso con la transformación e innovación, frente a la globalización tecnológica y el impacto que esta trae frente a la capacitación docente y estudiante. De manera que, la filosofía se encuentra presente en los complejos virtuales para el desarrollo cognitivo por el hecho de que implica una confrontación entre el conocimiento a priori basado en la experiencia y la evolución tecnológica presente.

En respuesta a esto, las universidades latinoamericanas frente al reto de contribuir tecnológicamente en aras de a una sociedad equitativa y democrática en pro de una revolución tecnológica se muestra de carácter pasivo y sistematizado; dado a que las universidades forman individuos que adoptan posturas preestablecida de cara a un contexto social burocrático inflexible que denota poca participación, afianzando mecanismos conductuales que hacen vida en el planeta, olvidándose de un modelo de vida sostenibles y las oportunidades de progreso por culpa del colonialismo intelectual que adoctrina el conocimiento empírico bajo un modelo de aprendizaje condicionado del ser humano encajonándolo en un sistema de sumisión disfrazado de globalización.

El reto implica la implementación de estructuras interdisciplinarias que tiendan a acentuar la naturalización y la manera de representar y entender el mundo a través de la lupa organizacional liberal/occidental. Asumiéndola desde afuera, descentralizando los poderes que anteceden a las instituciones educativas universitarias. Para ello, es necesaria una revolución tecnológica que arrope a la colectividad estudiantil que reclama un futuro lleno de posibilidades y progreso. 


\section{Referencias}

Arias, F. (2006). El Proyecto de Investigación. Introducción a la Metodología Científica. 5ta. Edición, ISBN: 980-07-8529-9, 143 págs. Caracas, Venezuela: Editorial Episteme, C.A. Recuperado de: https://es.slideshare.net/vcorreabalza/fidias-g-arias-el-proyecto-deinvestigacin-5ta-edicin-edicin-edicin

Barrionuevo, M. (2005). John Locke (1632-1704). Su vida, su obra y pensamiento. Revista Iberoamericana de Educación, 36(7), 1-10, eISSN: 1681-5653. Recuperado de:

https://rieoei.org/RIE/article/view/2979

Briones, G. (2002a,b,c,d,e,f,g,h). Epistemología de las ciencias Sociales. ISBN: 958-9329-09-8, 233 págs. Bogotá, Colombia: ARFO Editores e Impresores Ltda. Recuperado de:

https://www.insumisos.com/lecturasinsumisas/Epistemologia\%20de\%2 Olas $\% 20$ ciencias $\% 20$ sociales.pdf

Bustos, A., \& Coll, C. (2010). Los entornos virtuales como espacios de enseñanza y aprendizaje. Una perspectiva psicoeducativa para su caracterización y análisis. Revista mexicana de investigación educativa, 15(44), 163-184, e-ISSN: 1405-6666. Recuperado de:

http://www.scielo.org.mx/scielo.php?script=sci arttext\&pid=S1405$66662010000100009 \& \operatorname{lng}=\mathrm{es} \&$ tIng $=\mathrm{es}$

Diccionario soviético de filosofía (1965). Definición de empirismo. Montevideo, Uruguay: Ediciones Pueblos Unidos. Recuperado de: http://www.biblioteca.org.ar/Libros/fe/empi4.htm

Flores, K., \& Bravo, M. (2012). Metodología PACIE en los ambientes virtuales de aprendizaje para el logro de un aprendizaje colaborativo. Diálogos educativos, 12(24), 3-17, e-ISSN: 0718-1310. Recuperado de: https://dialnet.unirioja.es/servlet/articulo?codigo $=4156135$ 
Flores, K., \& Bravo, M. (2011). La plataforma MOODLE como herramienta evaluativa en los entornos virtuales de aprendizaje (EVA). Una experiencia con usuarios en la UPEL-IPB. EDUCARE, 15(3), 76-101, e-ISSN: 2244-7296. Recuperado de:

http://revistas.upel.edu.ve/index.php/educare/article/view/582

Guanoluisa, L. (2016). Desarrollo de habilidades cognitivas a través de prácticas experimentales. Primera Edición, ISBN: 978-9942-14-0746, 72 págs. Quito, Ecuador: Proyecto Etnomatemática - Runayupay.

Lander, E. (1999). ¿Conocimiento para qué? ¿Conocimiento para quién? Reflexiones sobre la universidad y la geopolítica de los saberes hegemónicos. Estudios Latinoamicanos, 7(12-13), 25-46, e-ISSN: 24484946. Recuperado de:

http://www.revistas.unam.mx/index.php/rel/article/view/52369

Londoño, C. (2008a,b,c,d,e). Avatares del constructivismo: de Kans a Piage. Revista Historia de la Educación Latinoamericana, (10), 73-96, e-ISSN: 0122-7238. Recuperado de:

http://www.redalyc.org/pdf/869/86901006.pdf

Mestre, U., Fonseca, J., \& Valdés, P. (2007). Entornos virtuales de enseñanza aprendizaje. ISBN: 978-959-16-0637-2, 78 págs. Cuba: Editorial Universitaria. Recuperado de:

http://libros.metabiblioteca.org/bitstream/001/251/8/978-959-16-06372.pdf

Raynaudo, G., \& Peralta, O. (2017). Cambio conceptual: una mirada desde las teorías de Piaget y Vygotsky. Liberabit, 23(1), 110-122, e-ISSN: 1729-4827. Recuperado de:

http://ojs3.revistaliberabit.com/index.php/Liberabit/article/view/56

Serrano, J., \& Pons, R. (2011). El constructivismo hoy: enfoques constructivistas en educación. REDIE. Revista Electrónica de Investigación Educativa, 13(1), 1-27, e-ISSN: 1607-4041. Recuperado 
de: https://www.redalyc.org/pdf/155/15519374001.pdf

Rojas, N., Pérez, F., Torres, I., \& Peláez, E. (2014). Las aulas virtuales: una opción para el desarrollo de la Educación Médica. EDUMECENTRO, 6(2), 231-247, e-ISSN: 2077-2874. Recuperado de: http://scielo.sld.cu/pdf/edu/v6n2/edu16214.pdf

Manriquez, J. (2016). Criterios de calidad de los EVA. Centro de Mejoramiento Docente. Copiapó, Chile: Vicerrectoría Académica de la Universidad de Atacama. Recuperado de:

http://www.cmd.uda.cl/index.php?option=com content\&view=article\&id $=433 \&$ Itemid $=388$

Uribe, P. (2017). Modalidad e-learning: espacio de autogestión para el aprendizaje en estudiantes en formación inicial docente de pedagogía en Chile. En P J., Sánchez-Rodríguez, J. y Sánchez-Rivas, E. (Edit.). Innovación docente y uso de las TIC en educación. Málaga, España: UMA Editorial. Recuperado de:

http://www.enriquesanchezrivas.es/congresotic/archivos/Universidad/U ribe Montanares.pdf

Zamudio, A., \& Castorina, J. (2017). La cuestión del a priori: de Kant a Piaget. Schème - Revista Eletrônica de Psicologia e Epistemologia Genéticas, 8(2), 04-27, e-ISSN: 1984-1655. Recuperado de: http://www2.marilia.unesp.br/revistas/index.php/scheme/article/view/66 $\underline{38}$ 


\section{Jurisbel Andreina Pérez Jiménez}

e-mail: jurisbel357@hotmail.com

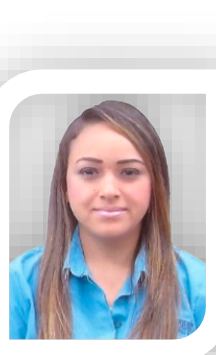

Nacida en el Municipio Cabimas, Estado Zulia, Venezuela, el 6 de febrero del año 1989. Licenciada en Educación Mención Informática de la Universidad del Zulia en el año 2010; Magíster Scientiarum en Informática Educativa de la Universidad Privada Dr. Rafael Belloso Chacín (URBE, 2013); cuento con Diplomados en Experto en Medios y Periodismo Digital (2017); Experto en Comercio Electrónico (2016); y Experto en Procesos E-learning (2013); en la Fundación para la actualización tecnológica de Latinoamérica (FATLA). Actualmente trabajo para la Alcaldía Bolivariana de Cabimas como operador de informática II. 


\section{Jurismar Ivana Pérez Jiménez}

e-mail: jurismarperez@gmail.com

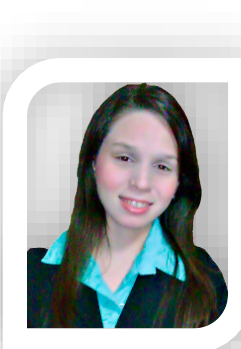

Nacida en el Municipio Cabimas, Estado Zulia, Venezuela, el 26 de junio del año 1991. Licenciada en Educación Mención Informática de la Universidad del Zulia en el año 2013; Magister Scientiarum en Gerencia de Recursos Humanos, egresada de la Universidad Nacional Experimental "Rafael María Baralt", en el año 2016. Actualmente trabajo en la E.T.C.R. "Hermágoras Chávez", soy Coordinadora, tutora y asesora de varios trabajos de Pasantías de este nivel. 


\section{Blanca Leticia Caldera de Sánchez}

e-mail: blancacaldera@hotmail.com

Nacida en el Municipio Cabimas, Estado Zulia,

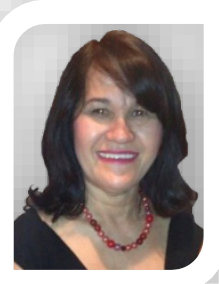

Venezuela, el 13 de septiembre del año 1953. Médico especialista en Pediatría y Puericultura, egresada de la Universidad del Zulia en el año 1980; Docente universitario de la asignatura Clínica Pediátrica en categoría agregado con dedicación a medio tiempo, en el núcleo Cabimas. Actualmente soy Coordinadora de Clínica Pediátrica Unidad Docente Cabimas, en el Hospital General de Cabimas "Dr. Adolfo D'Empaire". 
Nacida en el Municipio Cabimas, Estado Zulia,

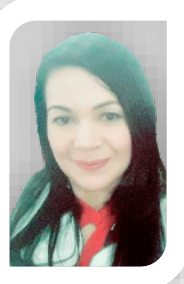
Venezuela, el 23 de junio del año 1976. Ingeniero en Sistemas, egresada del Instituto Universitario Politécnico "Santiago Mariño" de Cabimas en el año 1999; Magister Scientiarum en Informática Educativa (2010); y Doctora en Ciencias de la Educación de la Universidad Dr. Rafael Belloso Chacín (URBE, 2013). Actualmente soy docente titular de la Universidad del Zulia, Núcleo Costa Oriental del Lago. 